\title{
Numerical studies using finite different method for viscous dissipation and thermal radiation effects on the slip flow and heat transfer due to a stretching sheet embedded in a porous medium with variable thickness and variable thermal conductivity
}

\author{
A. Eid ${ }^{1}$ and M. Mohamed Khader ${ }^{2,3}$ \\ ${ }^{1}$ Department of Physics, College of Science, Al-Imam Mohammad Ibn Saud Islamic University (IMSIU), Saudi Arabia \\ ${ }^{2}$ Department of Mathematics and Statistics, College of Science, Al-Imam Mohammad Ibn Saud Islamic University (IMSIU), Saudi \\ Arabia \\ ${ }^{3}$ Department of Mathematics, Faculty of Science, Benha University Riyadh, Saudi Arabia and Giza, Egypt
}

Received: 7 November 2015, Revised: 15 November 2015, Accepted: 19 November 2015

Published online: 1 January 2016

\begin{abstract}
The aim of this paper is to study a numerical solution for the slip flow and heat transfer of a Newtonian fluid due to an impermeable stretching sheet which embedded in a porous medium with a power law surface velocity and variable thickness in the presence of thermal radiation, viscous dissipation and slip velocity effects. The flow is caused by a non-linear stretching of a sheet. Thermal conductivity of the fluid is assumed to vary linearly with temperature. The governing PDEs are transformed into a set of coupled non-linear ODEs by using appropriate boundary conditions for various physical parameters. The resulting system of ODEs is solved numerically using finite difference method (FDM). The effects of the porous parameter, the wall thickness parameter, the radiation parameter, the Eckert number, the slip velocity parameter, the thermal conductivity parameter and the Prandtl number on the flow and temperature profiles are presented. Moreover, the local skin-friction and Nusselt numbers are presented. Comparison of obtained numerical results is made with previously published results in some special cases, and excellent agreement is noted. The results attained in this paper confirm the idea that FDM is powerful mathematical tool and it can be applied to a large class of linear and nonlinear problems arising in different fields of science and engineering.
\end{abstract}

Keywords: Newtonian fluid, stretching sheet, slip velocity, variable thermal conductivity, thermal radiation, variable thickness, finite difference method.

\section{Introduction}

The study of flow and heat transfer of a Newtonian fluid over a stretching surface issuing from slit has gained considerable attention of many researchers due to its importance in many industrial applications, such as, extraction of polymer sheet, wire drawing, paper production, glass-fiber production, hot rolling, solidification of liquid crystals, petroleum production, continuous cooling and fibers spinning, exotic lubricants and suspension solutions. Much work on the boundary-layer Newtonian fluids has been carried out both experimentally and theoretically. Crane [1] was the first one who studied the stretching problem taking into account the fluid flow over a linearly stretched surface. There has been a great deal of the work done on Newtonian fluid flow and heat transfer over a stretching surface, but only a few recent studies are cited here. Gupta and Gupta [2] analyzed the stretching problem with a constant surface temperature, while Soundalgekar and Ramana [3] have investigated the constant surface velocity case with a power-law temperature 
variation. Grubka and Bobba [4] have analyzed the stretching problem for a surface moving with a linear velocity and with a variable surface temperature. Chen and Char [5] investigated the heat transfer characteristics over a continuous stretching sheet with variable surface temperature. Using the homotopy analysis method, series solutions were obtained by Hayat et al. [6] for the stretching sheet problem with mixed convection.

Despite the practical importance of the flow in a porous medium, all the above-mentioned works do not however consider the situations where the flow in fluid-saturated porous media arise. The study of the flow in fluid-saturated porous media due to a stretching sheet is important in engineering problems, such as the design of building components for energy consideration, soil science, mechanical engineering, control of pollutant spread in groundwater, thermal insulation systems, compact heat exchangers, solar power collectors and food industries. Because of such important practical applications, many investigators have modeled the behavior of a boundary layer flow embedded in a porous medium. Cheng and Minkowycz [7] studied the problem of free convection about a vertical impermeable flat plate in a Darcy porous medium. Elbashbeshy and Bazid [8] studied flow and heat transfer in a porous medium over a stretching surface with internal heat generation and suction/blowing when the surface is held at a constant temperature. Cortell [9] has presented an analytical solution of the problem considered by Elbashbeshy and Bazid [8] considering the constant surface temperature and prescribed surface temperature. Recently, Hayat et al. [10] used homotopy analysis method to give analytic solution for flow through porous medium.

In all the previous investigations, the effects of radiation on the flow and heat transfer have not been provided. Radiative heat transfer flow is very important in manufacturing industries for the design of reliable equipments, nuclear plants, gas turbines and various propulsion devices for aircraft, missiles, satellites and space vehicles. Also, the effect of thermal radiation on the forced and free convection flows are important in the context of space technology and processes involving high temperature. Based on these applications, Hossain et al. ([11], [12]) and Elbashbeshy and Demain [13] have studied the thermal radiation of a gray fluid which is emitting and absorbing radiation in non-scattering medium. Abel and Mahesha [14] studied the effect of radiation in different situations. Recently, Battaler [15] has studied the effect of thermal radiation on the laminar boundary layer about a flat plate.

Historically, the study on boundary layer flows over a stretching sheet with variable thickness was studied by Fang et al. [16]. However, so far no attention has been given to the effects of the non-flatness on the stretching sheet problems considering a variable sheet thickness. The purpose of the present paper is to investigate the numerical solution for the variable thermal conductivity, slip velocity and viscous dissipation effects on the flow and heat transfer of a Newtonian fluid-saturated porous medium over a stretching sheet with variable thickness in the presence of thermal radiation.

In this article, we implement the numerical technique, implicit FDM to the search for the numerical solutions of the introduced problem. The FDM plays an important rule in recent researches in this field. It has been shown that this procedure is a powerful tool for solving various kinds of problems ([17]-[24]). This technique reduces the problem to a system of algebraic equations. In this work, we use Newton iteration method to solve the resulting system of algebraic equations. Khader and Megahed in [18] presented numerical simulation using FDM for the flow and heat transfer in a thin liquid film over an unsteady stretching sheet in a saturated porous medium in the presence of thermal radiation. Sweilam, et al. [20] used Crank-Nicolson FDM for solving time-fractional diffusion equation. Also, Beckett in [24] used FDM for solving boundary layer type equation. Many authors have pointed out that FDM can overcome the difficulties arising in the calculation of some numerical methods, such as, finite element method ([17]-[24]). 


\section{Formulation of the problem}

Consider a steady, two-dimensional boundary layer flow of an incompressible Newtonian fluid over a continuously impermeable stretching sheet embedded in a porous medium. The origin is located at a slit, through which the sheet (see Fig. 1.) is drawn through the fluid medium. The $x$-axis is chosen along the sheet and $y$-axis is taken normal to it. The stretching surface has the velocity $U_{w}=U_{0}(x+b)^{m}$, where $U_{0}$ is the reference velocity. We assume that the sheet is not flat in which it is specified as $y=A(x+b)^{\frac{1-m}{2}}$, where $A$ is a very small constant so that the sheet is sufficiently thin and $m$ is the velocity power index. We must observe that our problem is valid only for $m \neq 1$, because for $m=1$, the problem reduces to a flat sheet. Likewise, the fluid properties are assumed to be constant except for thermal conductivity variations in the temperature.

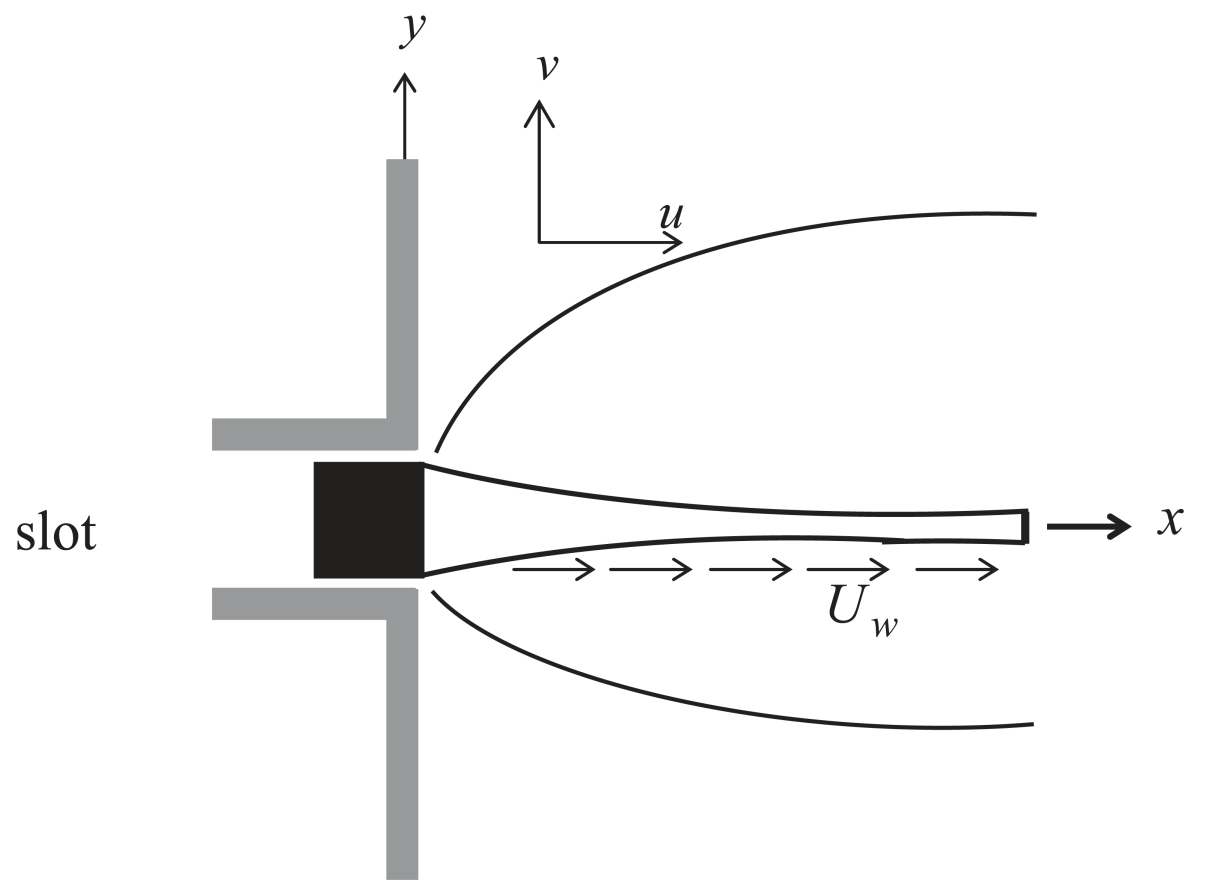

Fig. 1: Schematic of a stretching sheet with variable sheet thickness.

Making the usual boundary layer approximations for the Newtonian fluid, the steady two-dimensional boundary-layer equations taking into account the thermal radiation and viscous dissipation effects in the energy equation can be written as:

$$
\begin{gathered}
\frac{\partial u}{\partial x}+\frac{\partial v}{\partial y}=0 \\
u \frac{\partial u}{\partial x}+v \frac{\partial u}{\partial y}=v \frac{\partial^{2} u}{\partial y^{2}}-\frac{\mu}{\rho k} u \\
\rho c_{p}\left(u \frac{\partial T}{\partial x}+v \frac{\partial T}{\partial y}\right)=\frac{\partial}{\partial y}\left(\kappa \frac{\partial T}{\partial y}\right)-\frac{\partial q_{r}}{\partial y}+\mu\left(\frac{\partial u}{\partial y}\right)^{2}
\end{gathered}
$$


where $u$ and $v$ are the velocity components in the $x$ and $y$ directions, respectively. $\rho$ and $\kappa$ are the fluid density and the thermal conductivity, respectively. $T$ is the temperature of the fluid, $v$ is the fluid kinematic viscosity, $c_{p}$ is the specific heat at constant pressure, $\mu$ is the fluid viscosity, $k$ is the permeability of the porous medium and $q_{r}$ is the radiative heat flux.

The radiative heat flux $q_{r}$ is employed according to Rosseland approximation [25] such that

$$
q_{r}=-\frac{4 \sigma^{*}}{3 k^{*}} \frac{\partial T^{4}}{\partial y}
$$

where $\sigma^{*}=5.6697 \cdot 10^{-8} \mathrm{Wm}^{-2} \mathrm{~K}^{-4}$ is the Stefan-Boltzmann constant and $k^{*}$ is the mean absorption coefficient. Following Raptis [26], we assume that the temperature differences within the flow are sufficiently small such that $T^{4}$ may be expressed as a linear function of the temperature. Expanding $T^{4}$ in a Taylor series about $T_{\infty}$ and neglecting higher-order terms, we have

$$
T^{4} \cong 4 T_{\infty}^{3} T-3 T_{\infty}^{4}
$$

The physical and mathematical advantage of the Rosseland formula (6) consists of the fact that it can be combined with Fourier's second law of conduction to an effective conduction-radiation flux $q_{\text {eff }}$ in the form

$$
q_{e f f}=-\left(\kappa+\frac{16 \sigma^{*} T_{\infty}^{3}}{3 k^{*}}\right) \frac{\partial T}{\partial y}=-\kappa_{e f f} \frac{\partial T}{\partial y},
$$

where $\kappa_{\text {eff }}=\kappa+\frac{16 \sigma^{*} T_{\infty}^{3}}{3 k^{*}}$ is the effective thermal conductivity. So, the steady energy balance equation including the net contribution of the radiation emitted from the hot wall and absorbed in the colder fluid, takes the form

$$
\rho c_{p}\left(u \frac{\partial T}{\partial x}+v \frac{\partial T}{\partial y}\right)=\frac{\partial}{\partial y}\left(\kappa_{e f f} \frac{\partial T}{\partial y}\right)+\mu\left(\frac{\partial u}{\partial y}\right)^{2}
$$

To obtain similarity solutions, it is assumed that the permeability of the porous medium $k(x)$ is of the form $k(x)=$ $k_{0}(x+b)^{1-m}$ where $k_{0}$ is the permeability parameter. The boundary conditions can be written as

$$
\begin{aligned}
u\left(x, A(x+b)^{\frac{1-m}{2}}\right) & =U_{0}(x+b)^{m}+\lambda_{1}\left(\frac{\partial u}{\partial y}\right), \\
v\left(x, A(x+b)^{\frac{1-m}{2}}\right) & =0, \quad T\left(x, A(x+b)^{\frac{1-m}{2}}\right)=T_{w}, \\
u(x, \infty) & =0, \quad T(x, \infty)=T_{\infty},
\end{aligned}
$$

where $\lambda_{1}$ is the slip coefficient having dimension of length. To obtain similarity solutions, it is assumed that the slip coefficient $\lambda_{1}$ is of the form $\lambda_{1}=(x+b)^{\frac{1-m}{2}}$.

The mathematical analysis of the problem is simplified by introducing the following dimensionless coordinates

$$
\begin{gathered}
\eta=y \sqrt{U_{0}\left(\frac{m+1}{2}\right)\left(\frac{(x+b)^{m-1}}{v}\right)}, \psi(x, y)=\sqrt{v U_{0}\left(\frac{2}{m+1}\right)(x+b)^{m+1}} F(\eta), \\
\Theta(\eta)=\left(\frac{T-T_{\infty}}{T_{w}-T_{\infty}}\right),
\end{gathered}
$$


where $\eta$ is the similarity variable, $\psi(x, y)$ is the stream function which is defined in the classical form as $u=\partial \psi / \partial y$ and $v=-\partial \psi / \partial x$ and $\Theta(\eta)$ is the dimensionless temperature.

In this study, the equation for the dimensionless thermal conductivity $\kappa$ is generalized for the temperature dependence as follows ([27], [28])

$$
\kappa=\kappa_{\infty}(1+\varepsilon \Theta),
$$

where $\kappa_{\infty}$ is the ambient thermal conductivity and $\varepsilon$ is the thermal conductivity parameter.

Upon using these variables, the boundary layer governing equations (1)-(3) can be written in a non-dimensional following form as

$$
\begin{gathered}
F^{\prime \prime \prime}+F F^{\prime \prime}-\frac{2 m}{m+1} F^{\prime 2}-D F^{\prime}=0 \\
\left(\frac{1+R}{P r}\right)\left((1+\varepsilon \Theta) \Theta^{\prime \prime}+\varepsilon \Theta^{\prime 2}\right)+F \Theta^{\prime}+E c F^{\prime \prime 2}=0,
\end{gathered}
$$

where $D=\frac{2 v}{k_{0} U_{0}(m+1)}$ is the porous parameter, $P r=\frac{\mu c_{p}}{\kappa_{\infty}}$ is the Prandtl number, $E c=\frac{U_{w}^{2}}{c_{p}\left(T_{w}-T_{\infty}\right)}$ is the Eckert number and $R=\frac{16 \sigma^{*} T_{\infty}^{3}}{3 k^{*} \kappa_{\infty}}$ is the radiation parameter.

The transformed boundary conditions are

$$
\begin{gathered}
F(\alpha)=\alpha\left(\frac{1-m}{1+m}\right), \quad F^{\prime}(\alpha)=1+\lambda F^{\prime \prime}(\alpha), \quad \Theta(\alpha)=1, \\
F^{\prime}(\infty)=0, \quad \Theta(\infty)=0,
\end{gathered}
$$

where $\lambda=\frac{\sqrt{U_{0}(m+1)}}{\sqrt{2 v}}$ is the slip velocity parameter, $\alpha=A \sqrt{\frac{U_{0}(m+1)}{2 v}}$ is a parameter related to the thickness of the wall and $\eta=\alpha=A \sqrt{\frac{U_{0}(m+1)}{2 v}}$ indicates the plate surface. In order to facilitate the computation, we define $f(\zeta)=f(\eta-\alpha)=F(\eta)$ and $\theta(\zeta)=\theta(\eta-\alpha)=\Theta(\eta)$. The similarity equation and the associated boundary conditions become

$$
\begin{gathered}
f^{\prime \prime \prime}+f f^{\prime \prime}-\frac{2 m}{m+1} f^{\prime 2}-D f^{\prime}=0, \\
\left(\frac{1+R}{P r}\right)\left((1+\varepsilon \theta) \theta^{\prime \prime}+\varepsilon \theta^{\prime 2}\right)+f \theta^{\prime}+E c f^{\prime \prime 2}=0, \\
f(0)=\alpha\left(\frac{1-m}{1+m}\right), \quad f^{\prime}(0)=1+\lambda f^{\prime \prime}(0), \quad \theta(0)=1, \\
f^{\prime}(\infty)=0, \quad \theta(\infty)=0,
\end{gathered}
$$

where the prime denotes differentiation with respect to $\zeta$. Based on the variable transformation, the solution's domain will be fixed from 0 to $\infty$.

The physical quantities of primary interest are the local skin-friction coefficient $C f$ and the local Nusselt number $N u$ 
which are defined as

$$
C f=-2 \sqrt{\frac{m+1}{2}} \operatorname{Re}_{x}^{\frac{-1}{2}} f^{\prime \prime}(0), \quad N u=-\sqrt{\frac{m+1}{2}} \operatorname{Re}_{x}^{\frac{1}{2}} \theta^{\prime}(0),
$$

where $\operatorname{Re}_{x}=\frac{U_{w} X}{v}$ is the local Reynolds number and $X=x+b$.

\section{Solution procedure using FDM}

Our aim in this paper is to use the finite difference method to solve Eqs.(16)-(17) at the bounded domain $\left(0, \eta_{\infty}\right)$ with the boundary conditions (18)-(19). This method has been tested for accuracy and efficiency in solving different problems ([21], [23]).

To implement FDM, we use the transformation $f^{\prime}(\eta)=v(\eta)$ to rewrite Eqs.(16)-(17) in the following form

$$
\begin{gathered}
f^{\prime}-v=0 \\
v^{\prime \prime}+f v^{\prime}-\frac{2 m}{m+1} v^{2}-D v=0 \\
\left(\frac{1+R}{P r}\right)\left((1+\varepsilon \theta) \theta^{\prime \prime}+\varepsilon \theta^{\prime 2}\right)+f \theta^{\prime}+E c v^{\prime 2}=0,
\end{gathered}
$$

subject to the boundary conditions

$$
f(0)=\alpha\left(\frac{1-m}{1+m}\right), v(0)=1+\lambda v^{\prime}(0), \theta(0)=1, v\left(\eta_{\infty}\right)=0, \theta\left(\eta_{\infty}\right)=0 .
$$

In finite difference methods the space of solution's domain $\left(0, \eta_{\infty}\right)$ is discretized, $\eta_{\infty}=8$. We will use the following notations $\Delta \eta=\hbar>0$ to be the grid size in $\eta$-direction, $\Delta \eta=\eta_{\infty} / N$, with $\eta_{i}=i \hbar$ for $i=0,1, \ldots, N$. Define $f_{i}=f\left(\eta_{i}\right), v_{i}=v\left(\eta_{i}\right)$ and $\theta_{i}=\theta\left(\eta_{i}\right)$.

Let $F_{i}, V_{i}$ and $\Theta_{i}$ denote the numerical values of $f, v$ and $\theta$ at the $i^{t h}$ node, respectively. We take

$$
\begin{gathered}
\left.f^{\prime}\right|_{i}=\frac{f_{i+1}-f_{i-1}}{2 \hbar}+o\left(\hbar^{2}\right),\left.\quad v^{\prime}\right|_{i}=\frac{v_{i+1}-v_{i-1}}{2 \hbar}+o\left(\hbar^{2}\right), \\
\left.\theta^{\prime}\right|_{i}=\frac{\theta_{i+1}-\theta_{i-1}}{2 \hbar}+o\left(\hbar^{2}\right), \\
\left.v^{\prime \prime}\right|_{i}=\frac{v_{i+1}-2 v_{i}+v_{i-1}}{\hbar^{2}}+o\left(\hbar^{2}\right),\left.\theta^{\prime \prime}\right|_{i}=\frac{\theta_{i+1}-2 \theta_{i}+\theta_{i-1}}{\hbar^{2}}+o\left(\hbar^{2}\right) .
\end{gathered}
$$

The main step is that the system of ordinary differential equations (21)-(24) discretizes in space by using the FDM. To do this we substitute from (25)-(26) into (21)-(23) and neglecting the truncation errors, the resulting algebraic equations take the form, for $(i=1,2, \ldots, N-1)$.

$$
\begin{gathered}
F_{i+1}-F_{i-1}-2 \hbar V_{i}=0 \\
V_{i+1}-2 V_{i}+V_{i-1}+0.5 \hbar F_{i}\left(V_{i+1}-V_{i-1}\right)-\hbar^{2} V_{i}\left(\frac{2 m}{m+1} V_{i}+D\right)=0
\end{gathered}
$$




\begin{tabular}{|c|c|c|c|c|c|}
\hline$m$ & 9.00 & 7.00 & 5.00 & 3.00 & 2.00 \\
\hline$-f^{\prime \prime}(0)$ & 1.0589 & 1.0550 & 1.0486 & 1.0359 & 1.0234 \\
\hline Present work & 1.0590 & 1.0551 & 1.0487 & 1.0358 & 1.0235 \\
\hline \hline$m$ & 1.00 & 0.50 & 0.00 & $-1 / 3$ & -0.50 \\
\hline$-f^{\prime \prime}(0)$ & 1.0000 & 0.9799 & 0.9576 & 1.0000 & 1.1667 \\
\hline Present work & 1.0000 & 0.9798 & 0.9575 & 1.0000 & 1.1668 \\
\hline
\end{tabular}

Table 1: Comparison of the numerical value of $-f^{\prime \prime}(0)$, obtained by using FDM for $\alpha=0.5, \lambda=0$ and $D=0$ with Fang et al. [16].

\begin{tabular}{|c|c|c|c|c|c|}
\hline$m$ & 10.00 & 9.00 & 7.00 & 5.00 & 3.00 \\
\hline$-f^{\prime \prime}(0)$ & 1.1433 & 1.1404 & 1.1323 & 1.1186 & 1.0905 \\
\hline Present work & 1.1434 & 1.1404 & 1.1324 & 1.1185 & 1.0904 \\
\hline \hline$m$ & 1.00 & 0.50 & 0.00 & $-1 / 3$ & -0.50 \\
\hline$-f^{\prime \prime}(0)$ & 1.0000 & 0.9338 & 0.7843 & 0.5000 & 0.0833 \\
\hline Present work & 1.0000 & 0.9339 & 0.7842 & 0.5000 & 0.0834 \\
\hline
\end{tabular}

Table 2: Comparison of the numerical value of $-f^{\prime \prime}(0)$, obtained by using FDM for $\alpha=0.25, \lambda=0$ and $D=0$ with Fang et al. [16].

$$
\begin{array}{r}
(1+R)\left[\left(1+\varepsilon \Theta_{i}\right)\left(\Theta_{i+1}-2 \Theta_{i}+\Theta_{i-1}\right)+0.25 \varepsilon\left(\Theta_{i+1}-\Theta_{i-1}\right)^{2}\right] \\
+\operatorname{Pr}\left[0.5 \hbar F_{i}\left(\Theta_{i+1}-\Theta_{i-1}\right)+0.25 E c\left(V_{i+1}-V_{i-1}\right)^{2}\right]=0 .
\end{array}
$$

Also, the boundary conditions are

$$
\begin{aligned}
& F_{0}=\alpha\left(\frac{1-m}{1+m}\right), \quad V_{1}=\frac{\hbar}{\lambda}\left[-1+\left(1+\frac{\lambda}{\hbar}\right) V_{0}\right], \\
& \Theta_{0}=1, \quad V_{N}=0, \quad \Theta_{N}=0 .
\end{aligned}
$$

The system of Eqs.(27)-(30) is the numerical scheme of finite difference method and presents a non-linear system of algebraic equations in the variables $F_{i}, V_{i}$ and $\Theta_{i}$. In our calculation by using the Mathematica Package, we used the Newton iteration method with suitable initial solutions to obtain the numerical solution of this system.

\section{Results and discussion}

Tables 1 and 2 clearly reveal that present solution namely finite difference method shows excellent agreement with the existing solutions in the literature [16]. This analysis shows that FDM suits for the problems of boundary layer flow in fluid-saturated porous medium. This section provides the behavior of parameters involved in the expressions of heat transfer characteristics for the stretching sheet. Numerical evaluation for the solutions of this problem is performed and the results are illustrated graphically in Figs. 2-13. The study of flow in porous media is very important in approximating the shape of spherical particles or cylindrical fibers which better fit the model of permeability assumed for the analysis.

Effects of the porous parameter $D$ on velocity and temperature profiles are shown in Figs. 2 and 3, respectively. It is observed that the velocity decreases for increasing values of porous parameter. Furthermore, the momentum boundary layer thickness decreases as porous parameter $D$ increases. Fig. 3 elucidates that the fluid temperature enhance with an increase in the porous parameter. 


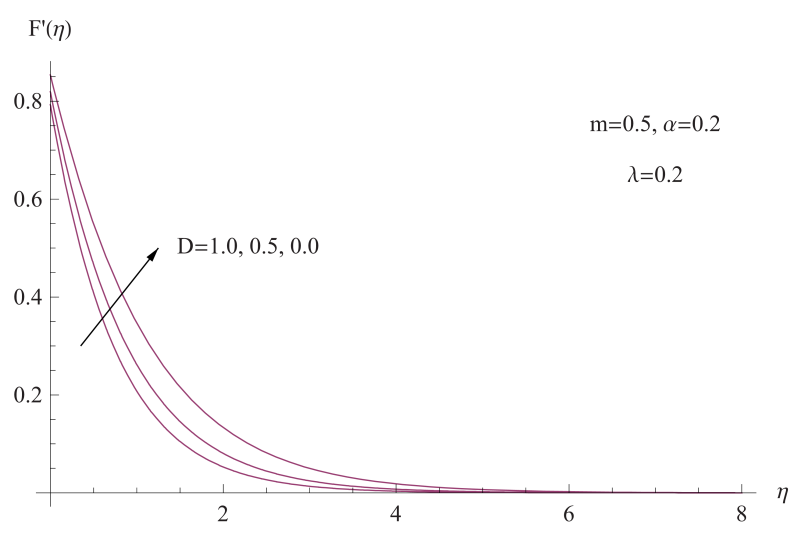

Fig. 2: The behavior of the velocity distribution for various values of $D$.

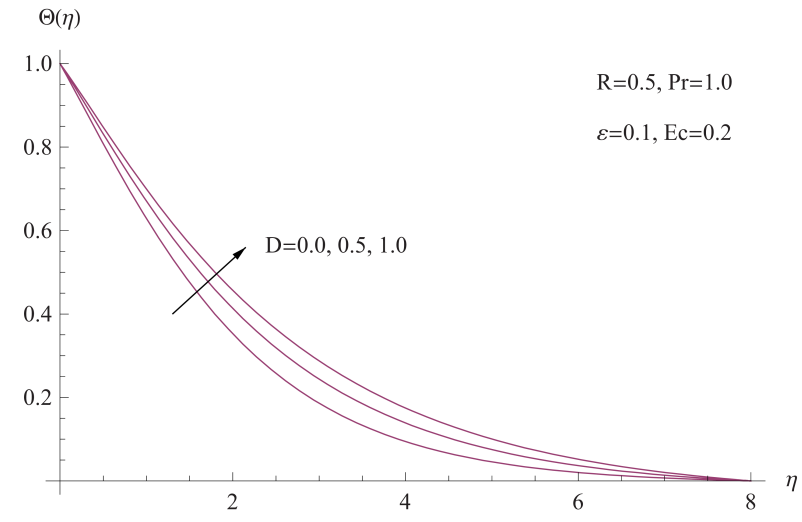

Fig. 3: The behavior of the temperature distribution for various values of $D$.

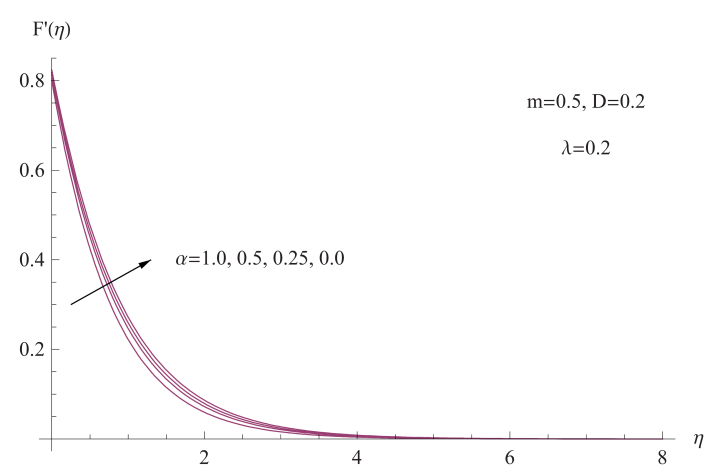

(a) The behavior of the velocity distribution for various values of $\alpha$ with $m=0.5$.

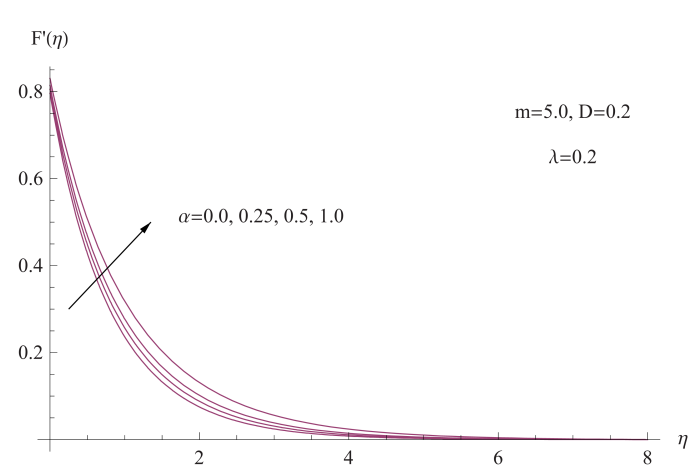

(b) The behavior of the velocity distribution for various values of $\alpha$ with $m=5$.

Fig. 4: The behavior of the velocity distribution for various values of $\alpha$.

The effects of wall thickness parameter on the fluid flow and the temperature distribution have been analyzed and the results are presented in Figs. 4-5. From Figs. 4 it is clear that the velocity at any point near to the plate decreases as the wall thickness parameter increases for $m<1$ and the reverse is true for $m>1$. Also, it is obvious from these figures that the thickness of the boundary layer becomes thinner for a higher value of $\alpha$ when $m<1$ and becomes thicker for a higher value of $\alpha$ when $m>1$.

Figs. 5 displays that the wall thickness parameter decreased the thickness of the thermal boundary layer and enhanced the rate of heat transfer for $m<1$ whereas reverse trend is observed as $m>1$. Physically, increasing the value of $\alpha$ when $m<1$ will decrease the flow velocity because under the variable wall thickness, not all the pulling force of the stretching sheet can be transmitted to the fluid causing a decrease for both friction between the fluid layers and temperature distribution for the fluid. But, when $m>1$ the velocity of the flow layers will increase causing an enhance for the friction force between this layers and thus increasing its temperature. Likewise, for a higher value of $\alpha$, the thermal boundary layer becomes thinner when $m<1$ compared with the case of $m>1$. 


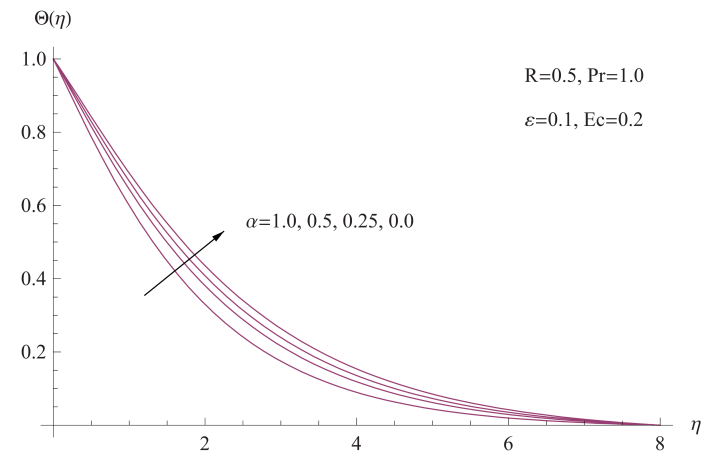

(a) The behavior of the temperature distribution for various values of $\alpha$ with $m=0.5$.

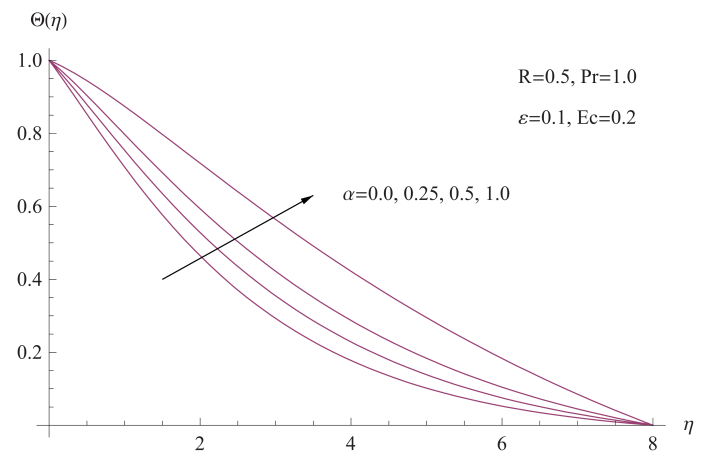

(b) The behavior of the temperature distribution for various values of $\alpha$ with $m=5$.

Fig. 5: The behavior of the temperature distribution for various values of $\alpha$.

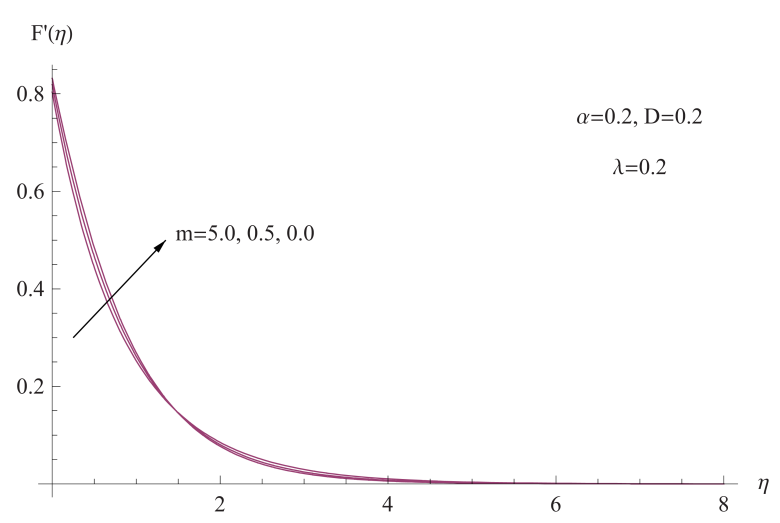

Fig. 6: The behavior of the velocity distribution for various values of $m$.

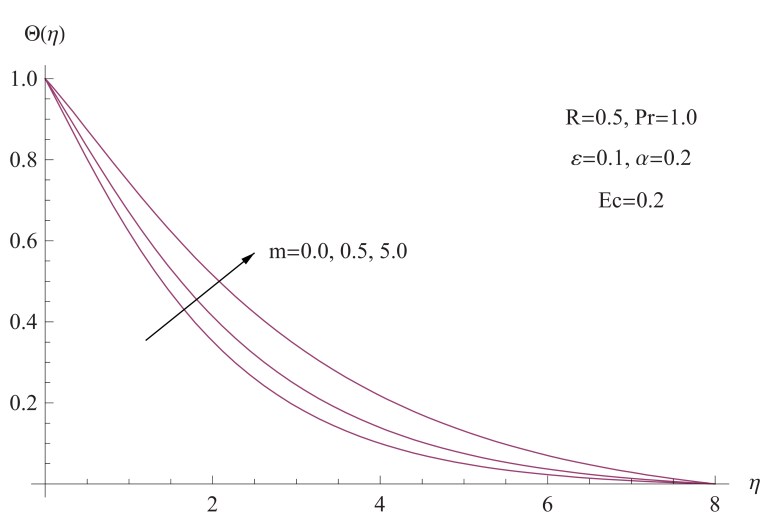

Fig. 7: The behavior of the temperature distribution for various values of $m$.

Fig. 6 shows that the velocity rises with a decrease in the values of the velocity power index $m$. This implies the momentum boundary thickness becomes thinner as the $m$ increases along the sheet and the reverse is true away from it.

Fig. 7 displays the influence of the velocity power index parameter $m$ on the temperature profiles. It is clearly seen from this figure that increasing the value of $m$ produces an increase in the temperature profiles. It further shows that the larger the value of $m$, the higher the magnitude of the thermal boundary thickness will be.

In Fig. 8, we have varied the thermal conductivity parameter $\varepsilon$ keeping the values of all other parameters fixed. Figure 8 reveals that the temperature profile as well as the thickness of the thermal boundary layer increase when $\varepsilon$ increases.

Figure 9 illustrates the effects of radiation parameter $R$ on the temperature profiles when other parameters held constant. It is depicted that the temperature field and the thermal boundary layer thickness increase with the increase in $R$.

It is observed from Fig. 10 that an increase in the Prandtl number results in decreases the heat transfer profiles. The reason is that increasing values of Prandtl number equivalent to decreasing the thermal conductivities and therefore heat 


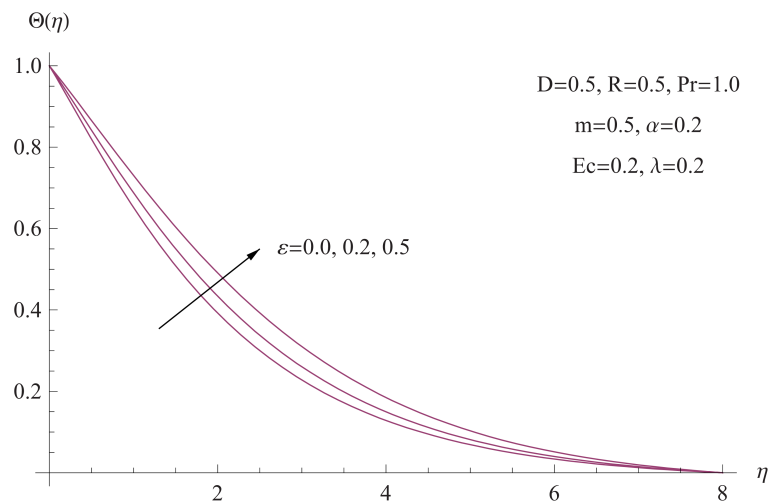

Fig. 8: The behavior of the temperature distribution for various values of $\varepsilon$.

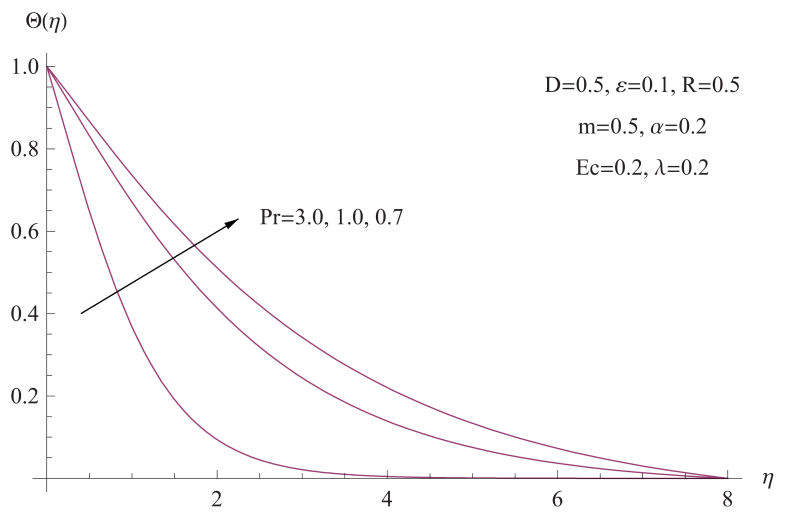

Fig. 10: The behavior of the temperature distribution for various values of $\mathrm{Pr}$.

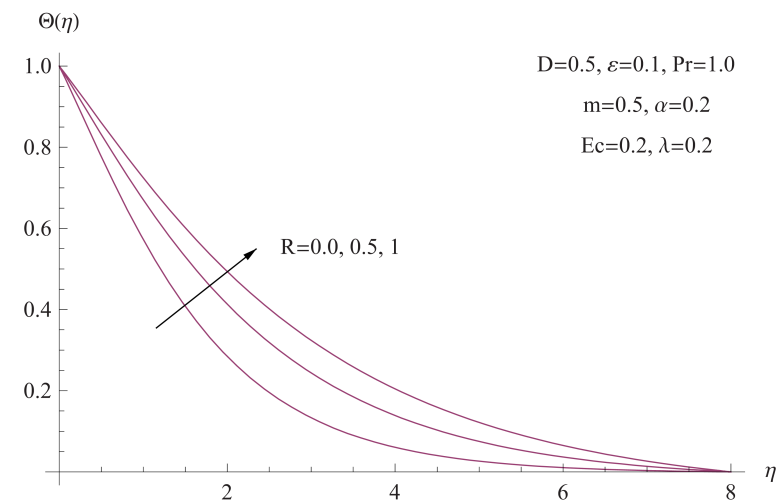

Fig. 9: The behavior of the temperature distribution for various values of $R$.

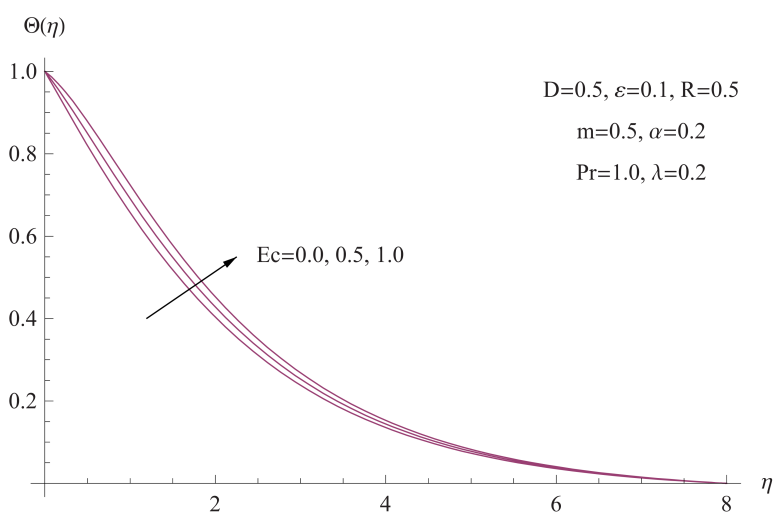

Fig. 11: The behavior of the temperature distribution for various values of $E c$.

is able to diffuse away from the heated sheet more rapidly. Hence in the case of increasing Prandtl number, the boundary layer is thinner and the heat transfer is reduced.

Fig. 11 is obtained by plotting the temperature distributions against variable $\eta$ for different values of Eckert number $E c$. Form this graph; it is clear that the temperature distribution increases with increase in the value of the Eckert number. Physically, this behavior is observed because in the presence of viscous dissipation, heat energy is stored in the fluid and there is significant more generation of heat along the sheet. Also, it is evident from this figure that the effect of viscous dissipation parameter is to enhance the thermal boundary layer thickness.

Figure 12 explain the variation of the slip velocity parameter $\lambda$ on the velocity profiles. From here we see that the velocity profiles decrease very rapidly throughout the fluid with the increase of slip velocity parameter $\lambda$. Likewise, this figure shows that the effect of increasing the slip velocity parameter $\lambda$ is to decrease the boundary layer thickness.

For various values of the slip velocity parameter $\lambda$, the profiles of the temperature distribution across the fluid is shown 


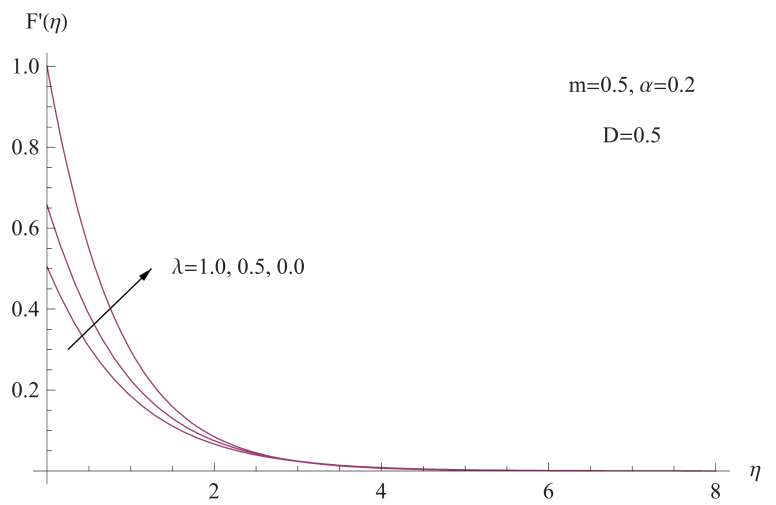

Fig. 12: The behavior of the velocity distribution for various values of $\lambda$.

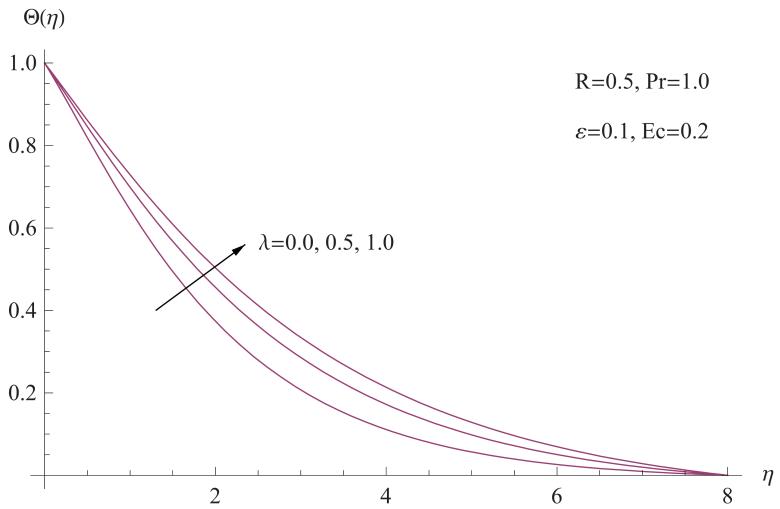

Fig. 13: The behavior of the temperature distribution for various values of $\lambda$.

\begin{tabular}{cccccccccc}
\hline$D$ & $\alpha$ & $m$ & $\varepsilon$ & $R$ & $P r$ & $E c$ & $\lambda$ & $-f^{\prime \prime}(0)$ & $-\theta(0)$ \\
\hline 0.0 & 0.2 & 0.5 & 0.1 & 0.5 & 1.0 & 0.2 & 0.2 & 0.731835 & 0.378228 \\
0.5 & 0.2 & 0.5 & 0.1 & 0.5 & 1.0 & 0.2 & 0.2 & 0.904128 & 0.324988 \\
1.0 & 0.2 & 0.5 & 0.1 & 0.5 & 1.0 & 0.2 & 0.2 & 1.036328 & 0.287806 \\
\hline 0.5 & 0.0 & 0.5 & 0.1 & 0.5 & 1.0 & 0.2 & 0.2 & 0.881918 & 0.301018 \\
0.5 & 0.25 & 0.5 & 0.1 & 0.5 & 1.0 & 0.2 & 0.2 & 0.909833 & 0.331014 \\
0.5 & 0.0 & 5.0 & 0.1 & 0.5 & 1.0 & 0.2 & 0.2 & 1.019823 & 0.276355 \\
0.5 & 0.25 & 5.0 & 0.1 & 0.5 & 1.0 & 0.2 & 0.2 & 0.973427 & 0.218568 \\
\hline 0.5 & 0.2 & 0.0 & 0.1 & 0.5 & 1.0 & 0.2 & 0.2 & 0.839815 & 0.394019 \\
0.5 & 0.2 & 0.5 & 0.1 & 0.5 & 1.0 & 0.2 & 0.2 & 0.904176 & 0.324954 \\
0.5 & 0.2 & 5.0 & 0.1 & 0.5 & 1.0 & 0.2 & 0.2 & 0.982522 & 0.229643 \\
\hline 0.5 & 0.2 & 0.5 & 0.0 & 0.5 & 1.0 & 0.2 & 0.2 & 0.904128 & 0.350602 \\
0.5 & 0.2 & 0.5 & 0.2 & 0.5 & 1.0 & 0.2 & 0.2 & 0.904193 & 0.303156 \\
0.5 & 0.2 & 0.5 & 0.5 & 0.5 & 1.0 & 0.2 & 0.2 & 0.904193 & 0.253845 \\
\hline 0.5 & 0.2 & 0.5 & 0.1 & 0.0 & 1.0 & 0.2 & 0.2 & 0.904175 & 0.432609 \\
0.5 & 0.2 & 0.5 & 0.1 & 0.5 & 1.0 & 0.2 & 0.2 & 0.904137 & 0.324942 \\
0.5 & 0.2 & 0.5 & 0.1 & 1.0 & 1.0 & 0.2 & 0.2 & 0.904193 & 0.268416 \\
\hline 0.5 & 0.2 & 0.5 & 0.1 & 0.5 & 0.7 & 0.2 & 0.2 & 0.904109 & 0.257244 \\
0.5 & 0.2 & 0.5 & 0.1 & 0.5 & 1.0 & 0.2 & 0.2 & 0.904189 & 0.324961 \\
0.5 & 0.2 & 0.5 & 0.1 & 0.5 & 3.0 & 0.2 & 0.2 & 0.904193 & 0.696949 \\
\hline 0.5 & 0.2 & 0.5 & 0.1 & 0.5 & 1.0 & 0.0 & 0.2 & 0.904155 & 0.366709 \\
0.5 & 0.2 & 0.5 & 0.1 & 0.5 & 1.0 & 0.5 & 0.2 & 0.904139 & 0.262122 \\
0.5 & 0.2 & 0.5 & 0.1 & 0.5 & 1.0 & 1.0 & 0.2 & 0.904144 & 0.157646 \\
\hline 0.5 & 0.2 & 0.5 & 0.1 & 0.5 & 1.0 & 0.2 & 0.0 & 1.168515 & 0.340438 \\
0.5 & 0.2 & 0.5 & 0.1 & 0.5 & 1.0 & 0.2 & 0.5 & 0.685216 & 0.303031 \\
0.5 & 0.2 & 0.5 & 0.1 & 0.5 & 1.0 & 0.2 & 1.0 & 0.495144 & 0.275558 \\
\hline
\end{tabular}

Table 3: Values of $-f^{\prime \prime}(0)$ and $-\theta(0)$ for various values of $D, \alpha, m, \varepsilon, R, E c, \lambda$ and $\operatorname{Pr}$.

in Figure 13. It is obvious that an increase in the slip velocity parameter $\lambda$ results in an increasing in both the temperature distribution throughout the thermal boundary layer and the thermal boundary layer thickness.

At this stage, we presented Table 3 in order to more fully characterize the behavior of the quantities of relevant physical interest like the local skin-friction coefficient and the local Nusselt number with changes in the porous parameter $D$, wall thickness parameter $\alpha$, the velocity power index parameter $m$, slip velocity parameter $\lambda$, Eckert number $E c$, the radiation parameter $R$, the Prandtl number $P r$ and thermal conductivity parameters $\varepsilon$ on the local skin friction coefficient and the 
local Nusselt number. It is noticed that increases in the wall thickness parameter leads to an increase in both the local skin-friction coefficient and the local Nusselt number when $m=0.5$ but the reverse is true for $m=5.0$. Likewise, the local Nusselt number is reduced but the skin-friction coefficient is increased with increasing for both values of porous parameter and velocity power index parameter. Also, an increase in the Prandtl number causes an increase in the local Nusselt number. This is because a fluid with larger Prandtl number possesses larger heat capacity, and hence intensifies the heat transfer. Moreover, it is observed that the values of the local Nusselt number decreases with increase in the Eckert number, the thermal conductivity parameter and the radiation parameter. Finally, the effect of slip velocity parameter is to decrease both the local Nusselt number and the local skin-friction coefficient.

\section{Conclusions}

Here we applied finite difference method to solve the resulting non-linear system of ordinary differential equations of the problem of slip flow and heat transfer in a quiescent Newtonian fluid flow caused solely by a stretching sheet which embedded in a porous medium with variable thickness, variable thermal conductivity, viscous dissipation and thermal radiation. The fluid thermal conductivity is assumed to vary as a linear function of temperature. comparison with previously published work was performed and the results were found to be in excellent agreement. Asystematic study on the effects of the various parameters on flow and heat transfer characteristics is carried out. It was found that the effect of increasing values of the porous parameter, the velocity power index parameter, thermal conductivity parameter, Eckert number and the radiation parameter reduce the local Nusselt number. On the other hand it was observed that the local Nusselt number increases as the Prandtl number and wall thickness parameter increases. Moreover, it is interesting to find that as the porous parameter, wall thickness parameter and the velocity power index parameter increases in magnitude, causes the fluid to slow down past the stretching sheet, the skin-fr iction coefficient increases in magnitude. Finally, both the local Nusselt number and the local skin-friction coefficient are decreases as the slip velocity parameter increases.

\section{Acknowledgements}

The authors thank Deanship of Academic Research, Al Imam Mohammad Ibn Saud Islamic University (IMSIU), Riyadh, KSA, for the financial support of the project number (351211).

\section{References}

[1] Crane, L. J., Flow past a stretching plate, Z. Angew. Math. Phys. 21, 645-647, (1970).

[2] Gupta, P. S. and Gupta, A. S., Heat and mass transfer on a stretching sheet with suction or blowing, Canadian J. Chem. Eng. 55(6), 744-746, (1979).

[3] Soundalgekar, V. M. and Ramana, T. V., Heat transfer past a continuous moving plate with variable temperature, Warme-Und Stoffuber tragung. 14, 91-93, (1980).

[4] Grubka, L. J. and Bobba, K. M., Heat transfer characteristics of a continuous stretching surface with variable temperature, J. Heat Transfer 107, 248-250, (1985).

[5] Chen, C. K and Char, M., Heat transfer on a continuous stretching surface with suction or blowing, J. Math. Anal. Appl. 35, 568-580, (1988).

[6] Hayat, T., Abbas, Z. and Javed, T., Mixed convection flow of a micropolar fluid over a non-linearly stretching sheet, Phys. Lett. A 372, 637-647, (2008). 
[7] Cheng, P. and Minkowycz, W. J., Free convection about a vertical flat plate embedded in a porous medium with application to heat transfer from a dike, J. Geophys. Res. 82, 2040-2044, (1977).

[8] Elbashbeshy, E. M. A. and Bazid, M. A. A., Heat transfer in a porous medium over a stretching surface with internal heat generation and suction or injection, Appl. Math. Comp. 158, 799-807, (2004).

[9] Cortell, R., Flow and heat transfer of a fluid through a porous medium over a stretching surface with internal heat generation/absorption and suction/blowing, Fluid Dyn. Res. 37, 231-245, (2005).

[10] Hayat, T., Abbas, Z. Pop, I. and Asghar, S., Effects of radiation and magnetic field on the mixed convection stagnation-point flow over a vertical stretching sheet in a porous medium Int. J. Heat Mass Transfer 53, 466-474, (2010).

[11] Hossain, M. A., Alim, M. A. and Rees, D., The effect of radiation on free convection from a porous vertical plate, Int. J. Heat Mass Transfer 42, 181-191, (1999).

[12] Hossain, M. A., Khanfer, K. and Vafai, K., The effect of radiation on free convection flow of fluid with variable viscosity from a porous vertical plate Int. J. Therm. Sci. 40, 115-124, (2001).

[13] Elbashbeshy, E. M. A. and Dimian, M. F., Effects of radiation on the flow and heat transfer over a wedge with variable viscosity, Appl. Math. Comp. 132, 445-454, (2002).

[14] Abel, M. S. and Mahesha, N., Heat transfer in MHD viscoelastic fluid flow over a stretching sheet with variable thermal conductivity, non-uniform heat source and radiation, Appl. Math. Modelling 32, 1965-1983, (2008).

[15] Bataller, R. C., Radiation effects in the Blasius flow, Appl. Math. Comput. 198, 333-338, (2008).

[16] Fang, T., Zhang, J. and Zhong, Y., Boundary layer flow over a stretching sheet with variable thickness, Appl. Math. Comput. 218, 7241-7252, (2012).

[17] Khader, M. M., On the numerical solutions for the fractional diffusion equation, Communications in Nonlinear Science and Numerical Simulation 16, 2535-2542, (2011).

[18] Khader, M. M., and Ahmed M. Megahed, Numerical simulation using the finite difference method for the flow and heat transfer in a thin liquid film over an unsteady stretching sheet in a saturated porous medium in the presence of thermal radiation, Journal of King Saud University: Engineering Sciences 25, 29-34, (2013).

[19] Sweilam, N. H., Khader, M. M. and Adel, M., Numerical simulation of fractional Cable equation of spiny neuronal dendrites, Journal of Advanced Research 1(1), 59-67, (2013).

[20] Sweilam, N. H., Khader, M. M. and Nagy, A. M., Numerical solution of two-sided space fractional wave equation using finite difference method, Journal of Computational and Applied Mathematics 235, 2832-2841, (2011).

[21] Butcher, J. C., Numerical Methods for Ordinary Differential Equations, John Wiley \& Sons (2003).

[22] Johnston, H. and Liu, J. G., Finite difference schemes for incompressible flow based on local pressure boundary conditions, Journal of Computational Physics 180, 120-154, (2002).

[23] Smith, G. D., Numerical Solution of Partial Differential Equations, Oxford University Press, New York, (1965).

[24] Beckett, P. M., Finite difference solution of boundary layer type equation, Int. J. Comput. Math. 14, 183-190, (1983).

[25] Raptis A., Flow of a micropolar fluid past a continuously moving plate by the presence of radiation, Int. J. Heat Mass Transfer 41, 2865-2866, (1998).

[26] Raptis A., Radiation and viscoelastic flow, Int. Commn. Heat Mass Transfer 26, 889-895, (1999).

[27] Chiam, T. C., Magnetohydrodynamic heat transfer over a non-isothermal stretching sheet, Acta Mech. 122, 169-179, (1997).

[28] Mahmoud, M. A. A. and Megahed, A. M., MHD flow and heat transfer in a non-Newtonian liquid film over an unsteady stretching sheet with variable fluid properties, Can. J. Phys. 87, 1065-1071, (2009). 